\title{
Lipopolysaccharide-induced podocyte injury is mediated by suppression of autophagy
}

\author{
XIAOFAN TAN ${ }^{1,2^{*}}$, YUANHAN CHEN $^{1 *}$, XINLING LIANG $^{1}$, CHUNPING YU $^{1}$, YUXIONG LAI $^{1}$,

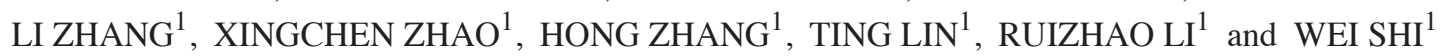 \\ ${ }^{1}$ Division of Nephrology, Guangdong General Hospital, Guangdong Academy of Medical Sciences, Guangzhou, \\ Guangdong 510080; ${ }^{2}$ Division of Nephrology, Zhongshan City People's Hospital/Zhongshan Hospital of \\ Sun Yat-sen University, Zhongshan, Guangdong 528400, P.R. China
}

Received March 20, 2015; Accepted March 11, 2016

DOI: $10.3892 / \mathrm{mmr} .2016 .5301$

\begin{abstract}
High-level autophagy has an important role in maintaining the stable state of podocytes. The present study explored the influence of lipopolysaccharide (LPS) on autophagic activity in podocytes and demonstrated its mechanistic involvement in LPS-induced injury. Conditionally immortalized podocytes were cultured in vitro and were treated with chloroquine (CQ), LPS, LPS+rapamycin or LPS+3-methyladenine (3-MA). The autophagic vesicles and endoplasmic reticulum were observed using transmission electron microscopy. The tandem mRFP-GFP-LC3 adenovirus was used to detect autophagosomes and autolysosomes. The expression levels of light chain 3-II (LC3 II), beclin-1, P62, CCAAT-enhancer-binding protein homologous protein (CHOP) and podocin were determined by western blot analysis. Autophagic vesicles were detected in podocytes under basic conditions. CQ was found to increase the protein levels of LC3 II in a time-dependent manner (2, 4 or $6 \mathrm{~h})$, confirming the high activity of autophagy in podocytes. Compared with the control group, LPS induced the expansion of the endoplasmic reticulum and high expression levels of CHOP, while decreasing the protein expression of podocin. Notably, podocytes treated with LPS showed decreases in LC3 II and beclin-1 levels and autophagosome/autolysosome numbers, which was accompanied by high P62 levels. Furthermore, the autophagy enhancer rapamycin reversed the downregulation of LC3 II and podocin, and the upregulation of CHOP induced by LPS, while the autophagy inhibitor 3-MA aggravated the effects of LPS. In conclusion, the present study demonstrated
\end{abstract}

Correspondence to: Professor Wei Shi, Division of Nephrology, Guangdong General Hospital, Guangdong Academy of Medical Sciences, 106 Zhongshan Road 2, Guangzhou, Guangdong 510080, P.R. China

E-mail: ssweicn@163.com

*Contributed equally

Key words: lipopolysaccharide, autophagy, podocyte injury that LPS inhibited podocyte autophagy, which contributed to LPS-induced injury of podocytes.

\section{Introduction}

Podocytes are among the structures of the glomerulus filtration barrier and are located around the glomerular basement membrane (GBM). As they have a similar morphology and structure to that of neurocytes, they are classified as a terminally differentiated cell type, in which the cell structures contain a cell body, preliminary processes and secondary processes (foot process). The intercellular junction of podocytes is formed in a staggered manner, based on the foot process (1). Differing from endothelial cells and the GBM, podocytes have an essential role in the selective glomerular filtration function. The abnormal cellular structure and function of podocytes can directly result in proteinuria, which may further develop into glomerulonephritis. Podocyte injury constitutes a common pathology in most glomerular diseases (2). In recent years, a large number of studies have focused on the mechanisms underlying podocyte injury and have led to a marked progression in this field $(3,4)$. In a clinical study, podocyte injury was targeted for the treatment of recurrent and refractory focal segmental glomerular sclerosis, ultimately resulting in partial or even complete remission (5). This study indicated great prospects for treatments targeting podocytes and suggested that further elucidation of podocyte injury mechanisms may be beneficial to the specific therapies for focal segmental glomerular sclerosis, membranous nephropathy, diabetic nephropathy, and so forth.

Lipopolysaccharide (LPS)-mediated podocyte injury is a commonly used model in studies on podocyte injury $(6,7)$. Previous studies have illustrated possible mechanisms of the LPS-induced injury in podocytes. For instance, LPS-induced activation of B7-1 results in the reorganization of vital slit diaphragm proteins (6), LPS-induced downregulation of the cell survival factor contributes to podocyte apoptosis (8) and LPS-induced activation of PTP1B leads to an increase in cell migration (9). A recent study by our group showed that LPS increased the activity of urokinase receptor-mediated beta 3 integrin signals to promote podocyte activity, thus resulting in cell injury $(10,11)$. Apart from these effects, the 
mechanisms of LPS-induced podocyte injury have remained elusive.

Autophagy, a lysosome-dependent bioactivity that results in the degradation of the cell's own biomacromolecules and organelles, has a significant role in the maintenance of cellular functions and resistance to exogenous stress (12). In contrast to other types of glomerular cells, normal podocytes demonstrate high levels of autophagic activity (13). Knockout of the autophagy-associated gene Atg5 was shown to decrease the high levels of autophagic activity in podocytes, resulting in the accumulation of oxidized and ubiquitinated proteins as well as endoplasmic reticulum (ER) stress. Furthermore, proteinuria, loss of podocytes and glomerular sclerosis were demonstrated in aged mice with Atg5 deficiencies (13). The essential role of autophagy in the maintenance of the bioactivities of podocytes is well known. However, whether changes in autophagic activity have a role during podocyte injury has remained elusive.

The present study generated an in vitro model of podocyte injury using LPS to assess the association between autophagic activity and podocyte injury.

\section{Materials and methods}

Reagents and equipment. Chloroquine, rapamycin and 3-methyladenine (3-MA) were all purchased from Sigma-Aldrich (St. Louis, MO, USA). LPS, as well as rabbit anti-podocin (cat. no. P0372) and rabbit anti-light chain (LC)3B (cat. no. L7543) antibodies were purchased from Sigma-Aldrich. Rabbit anti-Beclin-I (cat. no. 3738s), rabbit anti-P62 (cat. no. 5114s) and mouse-anti-CHOP (cat. no. 2895s) antibodies were purchased from Cell Signaling Technology, Inc. (Danvers, MA, USA). Mouse anti-glyceraldehyde-3-phosphate dehydrogenase (GAPDH) antibody was obtained from Bioworld Technology (St. Louis Park, MN, USA). Polyclonal horseradish peroxidase (HRP)-conjugated goat anti-mouse IgG (cat. no. 115-035-003) and goat anti-rabbit IgG (cat. no. 111-035-003) were purchased from Jackson ImmunoResearch Laboratories, Inc., West Grove, PA, USA). Red fluorescence protein - green fluorescence protein - LC3 double-tagged adenovirus (mRFP-GFP-LC3) was provided by Hanbio (Shanghai, China).

Podocyte culture. Differentiated podocytes are unable to replicate, and primary podocytes would result in rapid growth arrest when culturing. Conditionally immortalized mouse podocyte clones (MPCs) have overcome these difficulties, and conditionally immortalized MPC cells retain a differentiation potential similar to that of podocytes in vivo (14). With these characteristics, MPCs are widely used as a cell model in research focusing on human podocyte injury. Conditionally immortalized mouse podocytes were a gift from Professor J. Reiser of Rush University (Chicago, IL, USA). The recovered podocytes were cultured and passaged with Corning ${ }^{\circledR}$ cellgro ${ }^{\circledR}$ RPMI-1640 (Cellgro; Mediatech, Manassas, VA, USA) containing interferon (IFN)- $\gamma$ (10-100 U/ml; ProSpec, Tany Technogene Ltd., Rehovot, Israel), penicillin (100 U/ml), streptomycin $(100 \mu \mathrm{g} / \mathrm{ml})$ and $10 \%$ fetal bovine serum (FBS; Gibco $^{\circledR}$; Thermo Fisher Scientific, Waltham, MA, USA) at $33^{\circ} \mathrm{C}$ in a humidified atmosphere containing $5 \% \mathrm{CO}_{2}$ for 3-4 days. After proliferation was completed in the above medium, the podocytes were transferred into IFN- $\gamma$-free RPMI-1640 medium containing 5\% FBS and cultured at $37^{\circ} \mathrm{C}$ in a humidified atmosphere containing $5 \% \mathrm{CO}_{2}$ for 10-14 days for differentiation and maturation prior to use in the experiments of the present study.

Podocyte grouping and treatment. The matured podocytes were divided into seven groups as follows: i) CQ0h group/Control group (podocytes were cultured with normal medium); ii) CQ2h group [podocytes were cultured with chloroquine $(10 \mu \mathrm{M})$ for $2 \mathrm{~h}$ ]; iii) CQ4h group [podocytes were cultured with chloroquine $(10 \mu \mathrm{M})$ for $4 \mathrm{~h}]$; iv) CQ6h group [podocytes were cultured with chloroquine $(10 \mu \mathrm{M})$ for $6 \mathrm{~h}$ ]; v) LPS group [podocytes were cultured with LPS $(100 \mu \mathrm{g} / \mathrm{ml})$ for $24 \mathrm{~h}$ ]; vi) LPS + rapamycin group [podocytes were cultured with LPS $(100 \mu \mathrm{g} / \mathrm{ml})$ and rapamycin $(5 \mathrm{nM})$ for $24 \mathrm{~h}$; and vii) LPS + 3-MA group [podocytes were cultured with LPS $(100 \mu \mathrm{g} / \mathrm{ml})$ and $3-\mathrm{MA}(10 \mathrm{mM})$ for $24 \mathrm{~h}]$. The starting cell number for differentiating podocytes was $\sim 40,000$ per well on a 6-well plate with coverslips $(120,000$ per dish).

Western blot analysis. Following treatment with LPS and optionally with rapamycin or 3-MA, the podocytes were washed there times with cold phosphate-buffered saline (PBS) and then lysed on ice for $10 \mathrm{~min}$ using cell lysis buffer (Beyotime Institute of Biotechnology, Shanghai, China) containing proteinase inhibitor (phenylmethylsulfonyl fluoride; Guangzhou Genebase Bioscience Co., Ltd , Guangzhou, China) with scraping. The total protein was quantified using a bicinchoninic acid protein quantification kit (Thermo Fisher Scientific) After denaturation at $98^{\circ} \mathrm{C}$ for $10 \mathrm{~min}$, the protein ( $20 \mu \mathrm{g}$ per lane) was subjected to $12 \%$ sodium dodecyl sulfate polyacrylamide gel electrophoresis (Bio-Rad Laboratories, Inc., Hercules, CA, USA) and then transferred onto a nitrocellulose membrane (Millipore Corp., Boston, MA, USA) at $200 \mathrm{~mA}$. The blots were incubated with skimmed milk powder (5\%; YiLi, Inner Mongolia, China) for $1 \mathrm{~h}$ and then probed with the specific antibody at $4^{\circ} \mathrm{C}$ overnight. The blots were then incubated with the secondary antibody for $1 \mathrm{~h}$ and treated with an enhanced chemiluminescence (ECL) reagent (ECL Western Blotting substrate; Thermo Fisher Scientific). Images of the blots were captured on Kodak X-ray film (Eastman Kodak, Rochester, NY, USA). OPTIMAX X-ray film processor (Protec GmbH \& Co., Oberstenfeld, Germany) was used to capture the images, and scans of the images were subjected to quantitative analysis of the protein bands by densitometric analysis using ImageJ software (National Institutes of Health, Bethesda, MD, USA). The ratios of protein band intensities to GAPDH were determined and subsequently normalized to the control group or the CQ $0 \mathrm{~h}$ group.

Detection of autophagosomes and autolysosomes. Podocytes proliferating at $33^{\circ} \mathrm{C}$ were seeded into six-well plates containing coverslips coated with collagen type I (BD Biosciences, Franklin Lakes, NJ, USA) for 10-14 days (40,000 per well) and cultured until differentiation and maturation were achieved. The podocytes were inoculated with the MRFP-GFP-LC3 adenovirus at a concentration of $1 \times 10^{7} \mathrm{PFU} /$ well. The culture medium was replaced with fresh medium at $6 \mathrm{~h}$ post-inoculation and treatment [with LPS $(100 \mu \mathrm{g} / \mathrm{ml})$ intervention for $24 \mathrm{~h}$ ] was performed at $24 \mathrm{~h}$ post-inoculation. The cells were washed with 


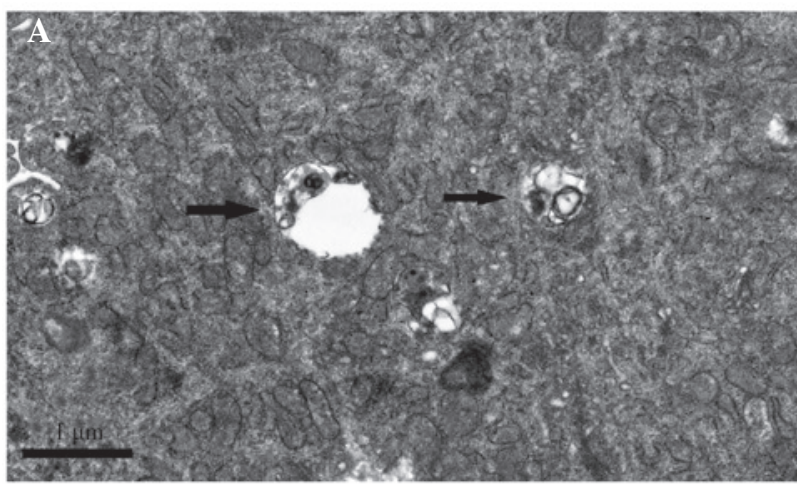

B

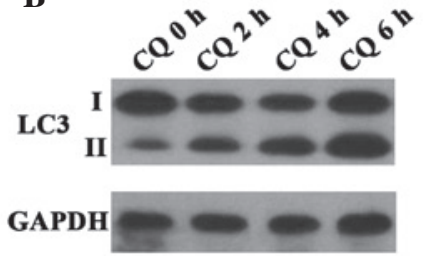

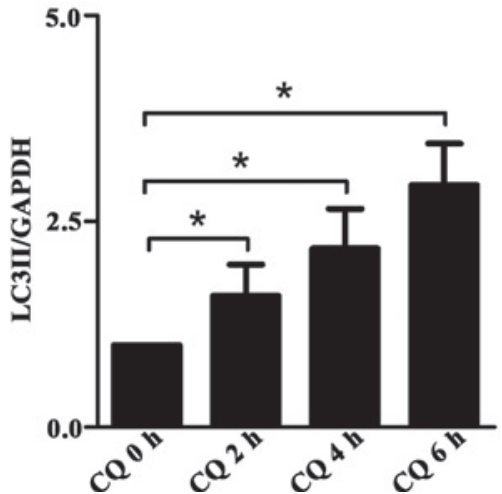

Figure 1. Detection of autophagic activity in podocytes. (A) Image of autophagosomes (indicated by arrows) acquired by transmission electron microscopy (magnification, x9,700). (B) The effects of CQ on LC3 I and II expression in podocytes were determined using western blotting. LC3 II expression was quantified by densitometric analysis. The ratio of LC3 II /GAPDH was normalized to CQ $0 \mathrm{~h}$. The value of the CQ $0 \mathrm{~h}$ group was 1 . Values are expressed as the mean \pm standard deviation $(n=3)$. * $\mathrm{P}<0.05$ vs. CQ 0 h. CQ, chloroquine; LC, light chain; GAPDH, glyceraldehyde-3-phosphate dehydrogenase.

PBS three times, fixed with 4\% paraformaldehyde (Guangzhou Whiga Technology Co., Ltd., Guangzhou, China) for $10 \mathrm{~min}$ and permeabilized with $0.5 \%$ Triton X-100 (Sigma-Aldrich) for $10 \mathrm{~min}$. The cells were stained with 4',6-diamidino-2-phenylindole (DAPI; Guangzhou Whiga Technology Co., Ltd.) for 6 min, mounted for anti-fluorescence quenching [using Antifade Mounting medium (Beyotime Institute of Biotechnology), a reagent that is able to achieve the effect of anti-fluorescence quenching], and observed using a Fluoview FV1000 laser scanning confocal microscope (Olympus, Tokyo, Japan). The mRFP-GFP-LC3 adenovirus-infected cells showed dual green and red fluorescence. LC3 II was recruited to the autophagosomal membranes, which was indicated by fluorescent spots. Red fluorescent spots indicated the autolysosome due to hydrolysis of GFP, whereas the autophagosome featured dual fluorescence, indicated by yellow spots. The numbers of autophagosomes and autolysosomes were recorded using Image $\mathbf{J}$ software (National Institutes of Health, Bethesda, MD, USA).

Observation of ER and autophagosomes by transmission electron microscopy (TEM). Following the indicated treatments, the cells were harvested in Eppendorf tubes $(1.5 \mathrm{ml})$, fixed with $2 \%$ glutaraldehyde (Sigma-Aldrich) at $4^{\circ} \mathrm{C}$ for $1 \mathrm{~h}$ and washed with cacodylate $(0.15 \mathrm{mM}$; Sigma-Aldrich) three times for $5 \mathrm{~min}$ each. The cells were then fixed with $1 \%$ osmium tetroxide (Sigma-Aldrich) at $4^{\circ} \mathrm{C}$ for $30 \mathrm{~min}$, washed with distilled water three times for $5 \mathrm{~min}$ each and treated with $1 \%$ uranyl acetate (Sigma-Aldrich) at $4^{\circ} \mathrm{C}$ for $30 \mathrm{~min}$. This step was followed by dehydration, embedding into epoxy resin (Sigma-Aldrich) for $12 \mathrm{~h}$ and polymerization at $60^{\circ} \mathrm{C}$. Cells prepared into resin embedded blocks were fixed in ultramicrotome (Leica EM UC6; Leica, Wetzlar, Germany) and then cut into ultrathin slices. The ultrathin slices were set into copper mesh. Images were captured by TEM using a JEM-100CX transmission electron microscope (Jeol, Tokyo, Japan) at magnifications of $\mathrm{x} 9,700$ or $\mathrm{x} 37,000$.

Statistical analysis. Values are expressed as the mean \pm standard deviation. Statistical analysis was performed using SPSS software, version 13.0 (SPSS, Inc., Chicago, IL, USA). Statistical significance was assessed using one-way ANOVA analysis of variance, followed by a least significant difference test for comparison between two groups. A two-tailed $\mathrm{P}<0.05$ was considered to indicate a statistically significant difference between values.

\section{Results}

Podocytes have high basal levels of autophagy. Autophagosomes were identified in normal podocytes using TEM (Fig. 1A). Chloroquine (CQ) was used to block the fusion of the autophagosomes and lysosomes in the podocytes for 2, 4 and $6 \mathrm{~h}$ to investigate autophagosome formation. Autophagy was evaluated based on an increase in LC3 II expression. The results demonstrated that CQ significantly increased LC3 II expression in a time-dependent manner (Fig. 1B), indicating that matured podocytes have a high basal level of autophagosome formation.

LPS induces podocyte injury. TEM observation indicated dilation of the ER in LPS-treated podocytes compared with those in the control group (Fig. 2A). In addition, western blot analysis revealed elevated protein expression levels of $\mathrm{CHOP}$ in the LPS-treated group compared with the control group $(\mathrm{P}=0.005)$ (Fig. 2B). By contrast, the expression of podocin was significantly inhibited in the LPS-treated group $(\mathrm{P}<0.001)$ (Fig. 2C).

LPS decreases autophagy in podocytes. Decreases in the protein levels of LC3 II $(\mathrm{P}=0.002)$ (Fig. 3A) and beclin-1 $(\mathrm{P}<0.001)$ (Fig. 3B), and an increase in the expression of P62 ( $\mathrm{P}=0.002)$ (Fig. 3C) were observed in the LPS-treated group compared with the control group. LC3 I also revealed a significant difference between the LPS-treated and the control groups, although this is not shown in Figure 3. Furthermore, laser scanning confocal microscopic observation of podocytes transfected with the adenovirus mRFP-GFP-LC3, which tagged the autophagosomes in yellow and the autolysosomes in red, showed a decreased ratio of autophagosomes to autolysosomes in the LPS-treated podocytes $(\mathrm{P}<0.001)$ (Fig. 4A and B).

Rapamycin inhibits LPS-induced autophagy suppression and podocyte injury. Treatment with rapamycin significantly 
A

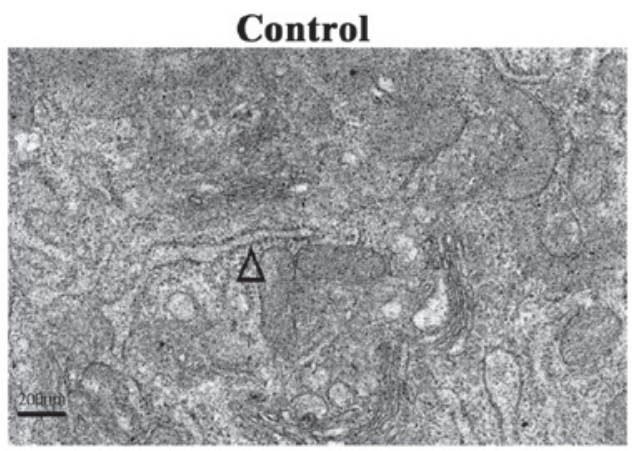

B

C
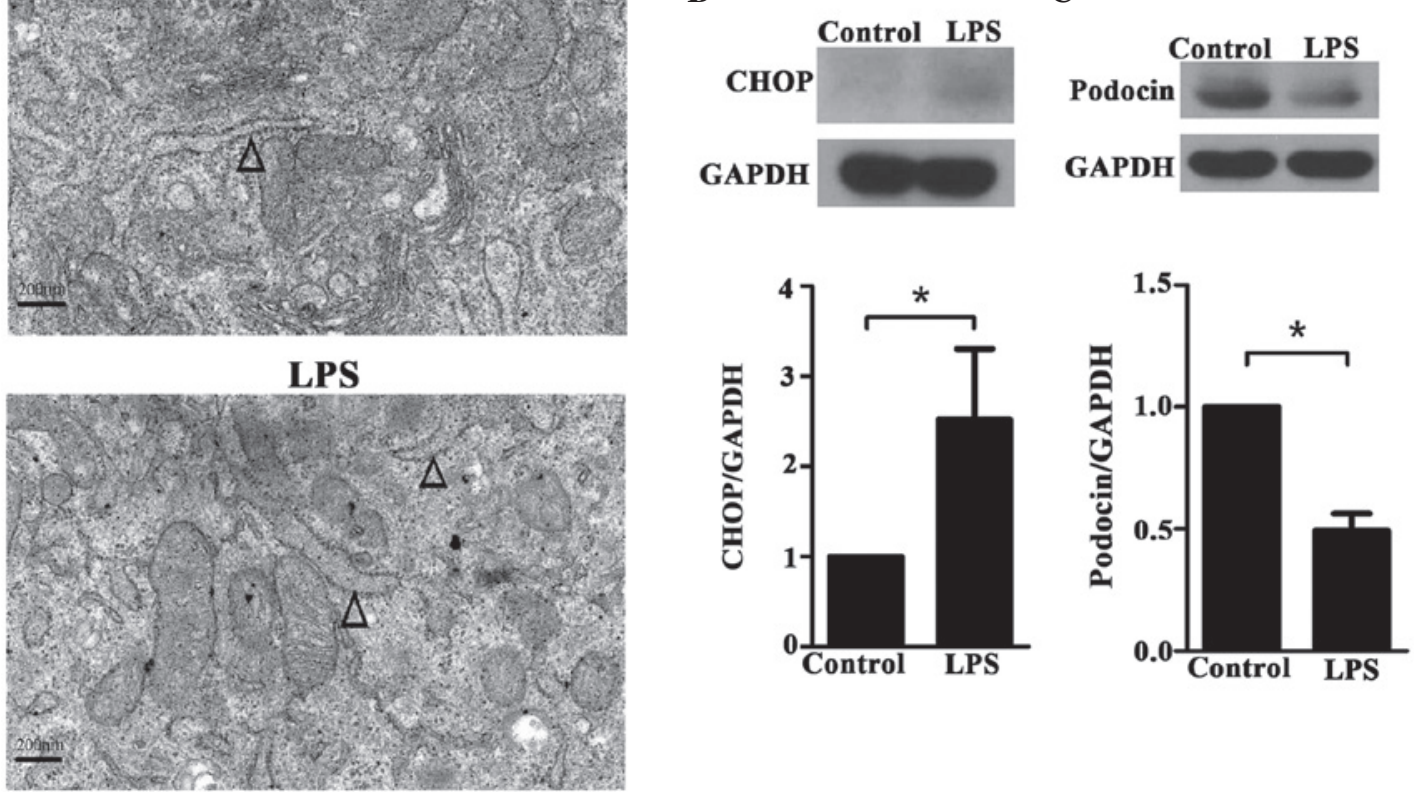

Figure 2. LPS-induced podocyte injury. (A) Transmission electron microscopy image (magnification, $\mathrm{x} 37,000$ ). The triangles indicate endoplasmic reticulum Effects of LPS on the levels of (B) CHOP and (C) slit diaphragm protein podocin determined by western blotting. Protein bands were quantified by densitometric analysis. The ratio of $\mathrm{CHOP} / \mathrm{GAPDH}$ and podocin/GAPDH were normalized to the control group. Values are expressed as the mean \pm standard deviation ( $\mathrm{n}=3$ ). ${ }^{*} \mathrm{P}<0.05$ vs. controls. LPS, lipopolysaccharide; GAPDH, glyceraldehyde-3-phosphate dehydrogenase; CHOP, CCAAT-enhancer-binding protein homologous protein.

inhibited LPS-induced decreases in LC3 II ( $\mathrm{P}=0.03$ ) (Fig. 5A). Even though rapamycin did not reverse the expression level of LC3I (Fig. 5A), this finding indicated that rapamycin partially restored autophagy in podocytes after LPS treatment. In addition, rapamycin attenuated LPS-induced decreases in the expression of podocin ( $\mathrm{P}=0.021)$ (Fig. 5B) and increases in the expression of $\mathrm{CHOP}(\mathrm{P}=0.013)$ (Fig. 5C), indicating that rapamycin inhibited LPS-induced podocyte injury.

3-MA aggravates LPS-induced autophagy suppression and cell injury in podocytes. The reduction of LC3 II expression in podocytes treated with LPS was shown to be aggravated by simultaneous treatment with 3-MA $(\mathrm{P}=0.022)$ (Fig. 6A), indicating that 3-MA contributed to LPS-induced autophagy suppression. In addition, a similar trend in the variation was observed in the expression level of LC3 I (Fig. 6A). In line with this, a further decrease in the levels of podocin $(\mathrm{P}=0.014)$ (Fig. 6B) and a further increase in $\mathrm{CHOP}$ expression $(\mathrm{P}=0.040)$ (Fig. 6C) by co-treatment with 3-MA compared with the group treated with LPS alone was observed.

\section{Discussion}

LPS has been used to establish models of renal injury, such as minimal change disease, focal segmental glomerulosclerosis (FSGS), acute kidney injury, and so forth (15-17). As illustrated above, some mechanisms of podocyte injury have been discovered during LPS treatment. Recently, some studies have demonstrated that a renoprotective role of autophagy in kidney tubules during LPS-inducing acute kidney injury $(17,18)$. Thus, it is hypothesized that autophagy plays a role in LPS-inducing podocyte injury. Autophagy is a quality control system that is involved in the maintenance of intracellular homeostasis. Its steps include autophagosome formation (engulfment of cytoplasmic proteins and injured organelles by double-membraned pre-autophagosomal structures), autolysosome formation (fusion of the autophagosomes and lysosomes) and autolysosomal degradation (generation of basal metabolism products) (19). Endogenous as well as exogenous stress can compel cells to recycle basal nutrition elements via autophagic degradation of abnormal intracellular components to maintain cellular structures and biofunctions. Furthermore, impairment of autophagy results in the accumulation of cell waste products and abnormal regeneration of long-lived proteins, which may result in the initiation of ER stress and cell apoptosis $(20,21)$. Therefore, normal autophagic activity is a necessity for long-lived, terminally differentiated cells, particularly neurocytes and podocytes.

In contrast to other cell types in the glomerulus, podocytes do not proliferate and must therefore attenuate injury stress by alternative means to cell regeneration or proliferation (1). Based on the specificity of their cell structure and biofunctions, podocytes have been found to be a significant target for glomerular injury (2). Therefore, it is indicated that podocytes face a particular challenge regarding homeostasis maintenance. Although the mechanisms of podocyte-associated pathogenesis of glomerular diseases remain largely elusive, recent studies have enhanced the current level of knowledge. The correlation between autophagy levels in podocytes and glomerular disease susceptibility has been reported by Hartleben et al (13). This study demonstrated that aged mice with autophagy-deficient podocytes suffered from proteinuria, which finally led to 
A

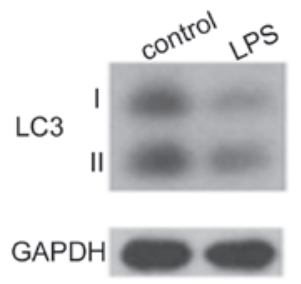

B
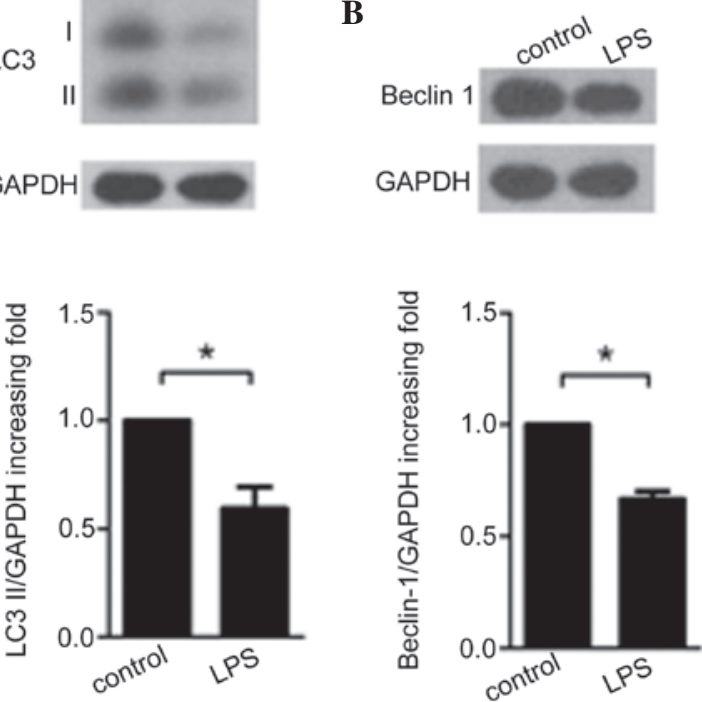
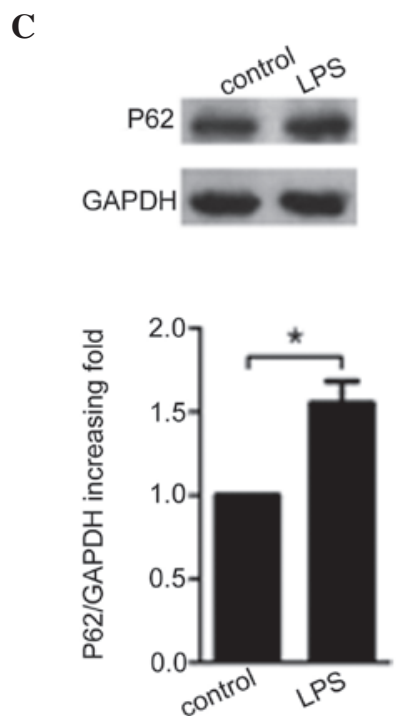

Figure 3. Effects of LPS on autophagy-associated proteins (A) LC3 I and II, (B) beclin-1 and (C) P62 in podocytes. Representative western blots are shown, and expression levels were quantified by gray value analysis of protein bands. The ratios of LC3 II/GAPDH, Beclin-1/GAPDH and P62/GAPDH were normalized to the control group. The value of the control group was 1 . Values are expressed as the mean \pm standard deviation. ${ }^{*} \mathrm{P}<0.05$ vs. control. LC, light chain; GAPDH, glyceraldehyde-3-phosphate dehydrogenase, LPS, lipopolysaccharide.
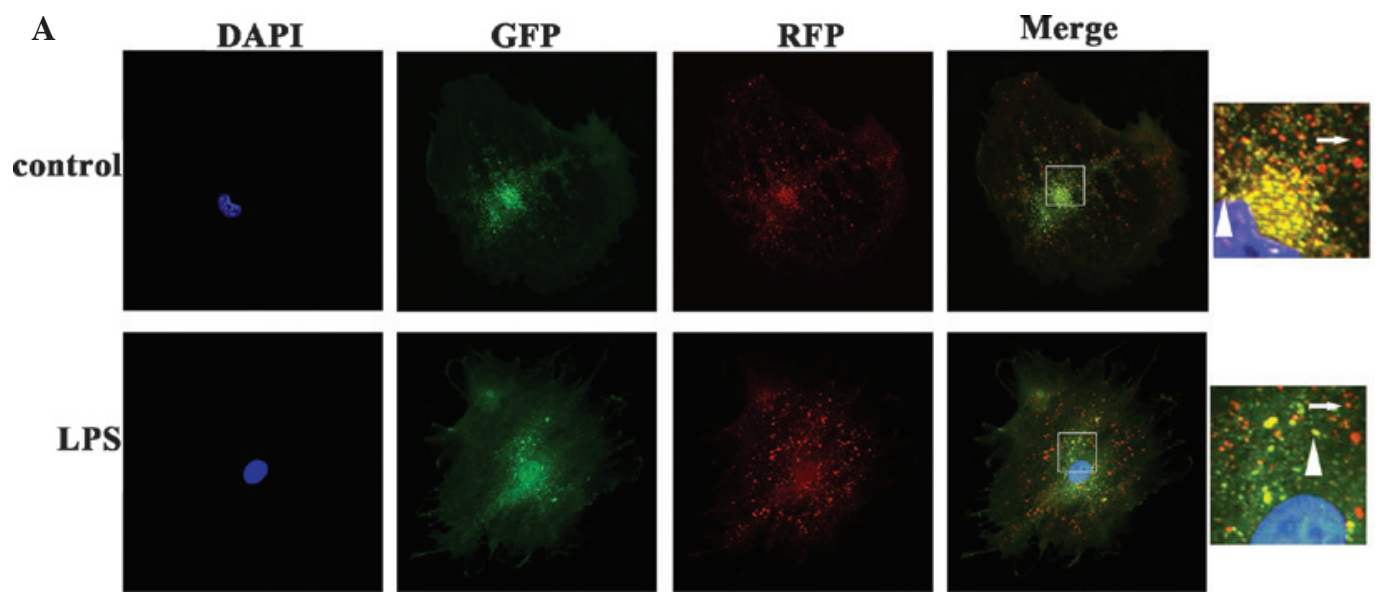

B

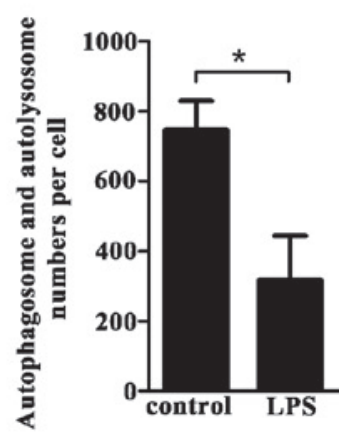

Figure 4. Effects of LPS on the amount of autophagosomes and autolysosomes in podocytes. (A) Laser scanning confocal microscopic images of autophagosomes (yellow) and autolysosomes (red) (magnification, x400). Magnified windows are shown (magnification, x16). (B) Quantified numbers of autphagosomes and autolysosomes per cell. Values are expressed as the mean \pm standard deviation $(\mathrm{n}=20)$. $\mathrm{P}<0.05$ vs. controls. LPS, lipopolysaccharide; GFP, green fluorescence protein; RFP, red fluorescence protein; DAPI, 4',6-diamidino-2-phenylindole.

glomerular sclerosis (13). This finding emphasized the significance of high levels of autophagy in the maintenance of cellular biofunctions. Recent clinicopathological studies analyzing renal biopsy specimens further supported that autophagic activity of podocytes has a critical protective role in renal injury (22-24). Glomeruli and in particular podocytes from patients with minimal changes in their disease status had higher levels of autophagic activity than glomeruli from patients with focal segmental glomerulosclerosis. Repeat renal biopsies of patients with minimal changes in their disease status enabled the tracking of podocyte autophagic activity and confirmed that patients maintaining high autophagic activity in their podocytes retained minimal change disease status, whereas patients with decreased autophagic activity progressed to focal segmental glomerulosclerosis (25). Consistent with the above studies, the present study detected a high basal level of autophagy in normal podocytes. In the present study, CQ treatment was employed to explore autophagic activity. CQ, an anti-malarial drug, can block the fusion of lysosomes and autophagosomes by increasing the $\mathrm{pH}$ value of the lysosomes. This process results in the abortive formation of the autolysosomes and the accumulation of newly generated autophagosomes (26). Microtubule-associated protein LC3, a mammalian homologue of yeast Atg8, exists in two forms: LC3-I and LC3-II. LC3-I is an abundant cytoplasmic protein that is cleaved and lipidated during initiation of autophagy (forming LC3-II), translocating to and associating with the autophagosome in a punctate pattern. Thus, LC3-II is considered as an autophagy biomarker. Based on the characteristics of CQ, the protein levels of LC3-II were determined at different CQ intervening time points to indirectly 

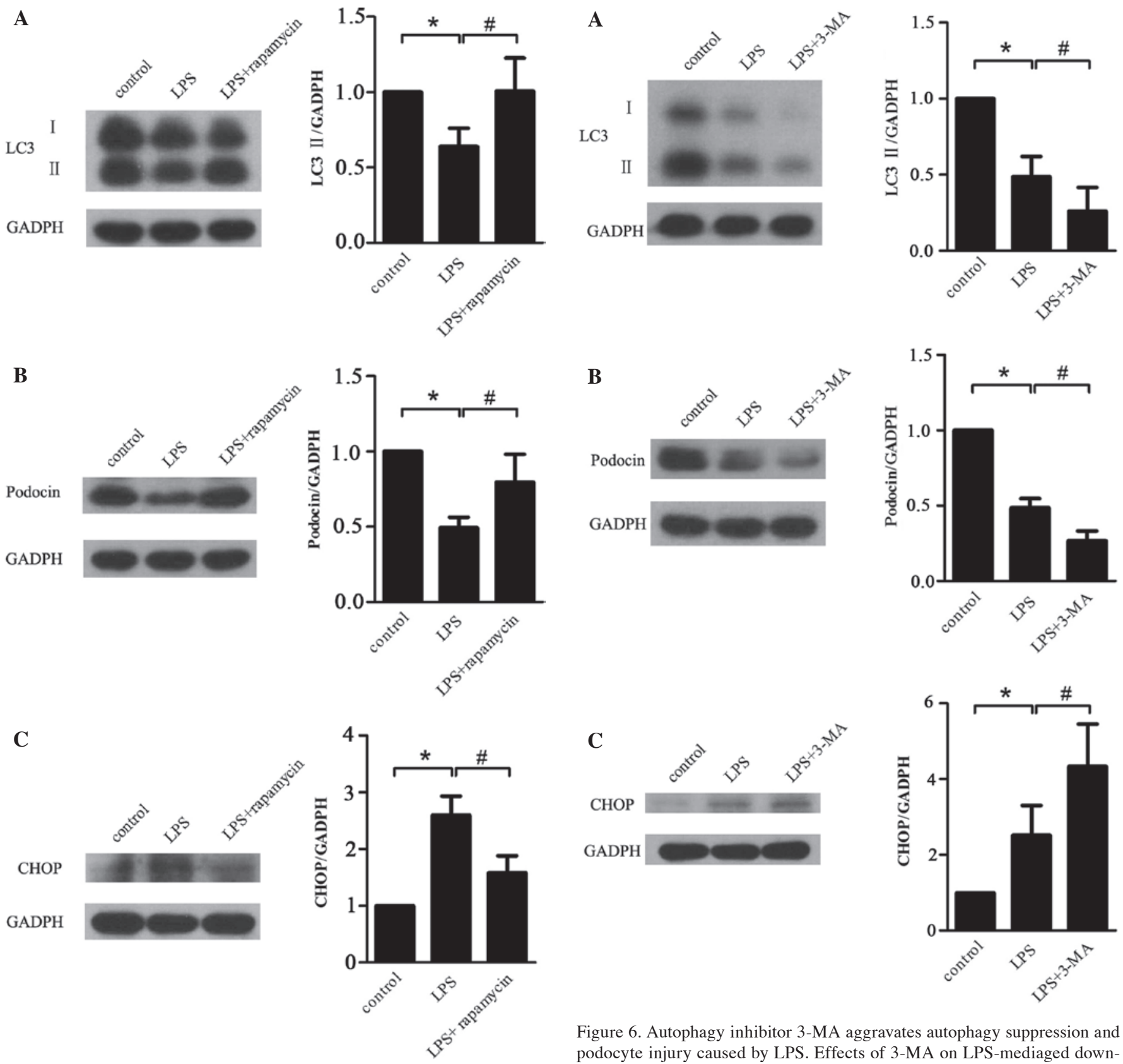

Figure 6. Autophagy inhibitor 3-MA aggravates autophagy suppression and podocyte injury caused by LPS. Effects of 3-MA on LPS-mediaged downregulation of (A) LC3 II and (B) podocin and (C) upregulation of CHOP. Representative western blots are shown, and expression levels were quantified by gray value analysis of protein bands. The ratios of LC3 II/GAPDH, podocin/GAPDH and CHOP/GAPDH were normalized to the control group. The value of the control group was 1 . Values are expressed as the mean \pm standard deviation ( $\mathrm{n}=3$ ). ${ }^{\text {"P }}<0.05$ vs. controls; ${ }^{~} \mathrm{P}<0.05$ vs. LPS+3-MA. LC, light chain; GAPDH, glyceraldehyde-3-phosphate dehydrogenase, LPS, lipopolysaccharide; CHOP, CCAAT-enhancer-binding protein homologous protein; 3-MA, 3-methyladenine. LPS+rapamycin. LC, light chain; GAPDH, glyceraldehyde-3-phosphate dehydrogenase, LPS, lipopolysaccharide; CHOP, CCAAT-enhancer-binding protein homologous protein.

indicate the level of autophagy activity. The results showed that LC3 II rapidly accumulated following CQ treatment, indicating a high level of autophagy in the podocytes. Furthermore, a decrease in the expression level of LC3-I was also observed in the LPS-treated group. This suggested that the inhibition of autophagy could involve two aspects: Downregulation of the expression of LC3-I and the conversion inhibition of LC3-I to LC3-II.

The results of the present study demonstrated that LC3 II and beclin-1 were downregulated in LPS-treated podocytes. P62 has been used in numerous studies as a protein marker to indicate autophagic activity. It is an adapter protein, which can bind to LC3 II and ubiquitinated aggregated proteins and facilitate the transportation of ubiquitinated proteins to the autophagosomes. Subsequently, P62 is degraded together with the ubiquitinated proteins in the autolysosomes (27). In the present study, P62 expression in podocytes was found to be significantly increased following LPS treatment. The 
dual-tagged adenovirus mRFP-GFP-LC3 has been previously used to detect autophagosomes (28), and was used to investigate the effects of LPS on autophagy in podocytes. In this system, the stability of mRFP can compensate for the instability of GFP in an acidic environment, enabling for the simultaneous detection of autophagosomes and autolysosomes. In the present study, the number of autophagosomes and autolysosomes was decreased by LPS. All of these findings indicated that LPS inhibited the high-level autophagic activity in podocytes.

Previous studies have reported that ER stress and abnormal slit diaphragm proteins have significant roles in the injury of podocytes and thus serve as known markers of podocyte injury (29). In the present study, LPS treatment resulted in ER dilation, increased expression levels of the ER stress-associated protein CHOP and a significant decrease in the a slit diaphragm protein podocin. To investigate the association between LPS-induced podocyte injury and autophagy, autophagy enhancer rapamycin and autophagy suppressor 3-MA were employed $(30,31)$. The results showed that rapamycin partly restored LPS-mediated decreases of autophagy, and attenuated LPS-induced ER stress and downregulation of podocin. By contrast, 3-MA aggravated the effects of LPS on autophagy and podocyte injury.

In spite of the findings of the present and previous studies, the underlying mechanisms of LPS-induced podocyte injury and inhibition of autophagy remain to be fully elucidated. The migration of autophagosomes and lysosomes depends on components of the cytoskeleton, such as microtubules (32), and it is known that LPS damages the skeletal systems of podocytes (6). Whether LPS affects autophagic activity of podocytes by damaging the skeletal system is worthy of further exploration.

In conclusion, the present study reported that LPS-induced podocyte injury is, at least in part, mediated via inhibition of autophagy. 3-MA-induced inhibition of autophagy significantly enhanced LPS-induced podocyte injury, while rapamycin-mediated restoration of autophagy attenuated the effects of LPS. The present study therefore provided a basis for the development of novel strategies for the treatment of glomerular diseases, such as the upregulation of autophagy under injury stress.

\section{Acknowledgements}

This study received financial support from the National Natural Science Foundation of China (81270784; 81470930) and the National Clinical Key Specialty Construction Projects.

\section{References}

1. Pavenstadt H, Kriz W and Kretzler M: Cell biology of the glomerular podocyte. Physiol Rev 83: 253-307, 2003.

2. Greka A and Mundel P: Cell biology and pathology of podocytes. Annu Rev Physiol 74: 299-323, 2012.

3. Gubler MC: Podocyte differentiation and hereditary proteinuria/nephrotic syndromes. J Am Soc Nephrol 14 Suppl 1: S22-S26, 2003.

4. Patrakka J and Tryggvason K: New insights into the role of podocytes in proteinuria. Nat Rev Nephrol 5: 463-468, 2009.
5. Yu CC, Fornoni A, Weins A, Hakroush S, Maiguel D, Sageshima J, Chen L, Ciancio G, Faridi MH, Behr D, et al: Abatacept in B7-1-positive proteinuric kidney disease. N Engl J Med 369: 2416-2423, 2013.

6. Reiser J, von Gersdorff G, Loos M, Oh J, Asanuma K, Giardino L, Rastaldi MP, Calvaresi N, Watanabe H, Schwarz K, et al: Induction of B7-1 in podocytes is associated with nephrotic syndrome. J Clin Invest 113: 1390-1397, 2004.

7. Wei C, Moller CC, Altintas MM, Li J, Schwarz K, Zacchigna S, Xie L, Henger A, Schmid H, Rastaldi MP, et al: Modification of kidney barrier function by the urokinase receptor. Nat Med 14: 55-63, 2008

8. Saurus P, Kuusela S, Lehtonen E, Hyvönen ME, Ristola M Fogarty CL, Tienari J, Lassenius MI, Forsblom C, Lehto M, et al: Podocyte apoptosis is prevented by blocking the Toll-like receptor pathway. Cell Death Dis 6: e1752, 2015.

9. Kumagai T, Baldwin C, Aoudjit L, Nezvitsky L, Robins R, Jiang R and Takano T: Protein tyrosine phosphatase 1B inhibition protects against podocyte injury and proteinuria. Am J Pathol 184: 2211-2224, 2014.

10. Zhang B, Shi W, Ma J, Sloan A, Faul C, Wei C, Reiser J, Yang Y, Liu S and Wang W: The calcineurin-NFAT pathway allows for urokinase receptor-mediated beta3 integrin signaling to cause podocyte injury. J Mol Med (Berl) 90: 1407-1420, 2012.

11. Zhang B, Xie S, Shi W and Yang Y: Amiloride off-target effect inhibits podocyte urokinase receptor expression and reduces proteinuria. Nephrol Dial Transplant 27: 1746-1755, 2012.

12. Mizushima N, Levine B, Cuervo AM and Klionsky DJ: Autophagy fights disease through cellular self-digestion. Nature 451: 1069-1075, 2008.

13. Hartleben B, Gödel M, Meyer-Schwesinger C, Liu S, Ulrich T, Köbler S, Wiech T, Grahammer F, Arnold SJ,Lindenmeyer MT, et al: Autophagy influences glomerular disease susceptibility and maintains podocyte homeostasis in aging mice. J Clin Invest 120: 1084-1096, 2010.

14. Mundel P, Reiser J, Zúñiga Mejía Borja A, Pavenstädt $H$, Davidson GR, Kriz W and Zeller R: Rearrangements of the cytoskeleton and cell contacts induce process formation during differentiation of conditionally immortalized mouse podocyte cell lines. Exp Cell Res 236: 248-58, 1997.

15. Srivastava T, Sharma M, Yew KH, Sharma R, Duncan RS, Saleem MA, McCarthy ET, Kats A, Cudmore PA, Alon US, et al: LPS and PAN-induced podocyte injury in an in vitro model of minimal change disease: changes in TLR profile. J Cell Commun Signal 7: 49-60, 2013

16. Reiser J, von Gersdorff G, Loos M, Oh J, Asanuma K, Giardino L, Rastaldi MP, Calvaresi N, Watanabe H, Schwarz K, et al: Induction of B7 1 in podocytes is associated with nephrotic syndrome. J Clin Invest 113: 1390-1397, 2004.

17. Howell GM, Gomez H, Collage RD, Loughran P, Zhang X, Escobar DA, Billiar TR, Zuckerbraun BS, Rosengart MR: Augmenting autophagy to treat acute kidney injury during endotoxemia in mice. PLoS One 8: e69520, 2013.

18. Mei S, Livingston M, Hao J, Li L, Mei C and Dong Z: Autophagy is activated to protect against endotoxic acute kidney injury. Sci Rep 6: 22171, 2016.

19. Klionsky DJ and Emr SD: Autophagy as a regulated pathway of cellular degradation. Science 290: 1717-1721, 2000.

20. Komatsu M, Waguri S, Chiba T, Murata S, Iwata J, Tanida I, Ueno T, Koike M, Uchiyama Y and Kominami E: Loss of autophagy in the central nervous system causes neurodegeneration in mice. Nature 441: 880-884, 2006.

21. Maiuri MC,Zalckvar E, Kimchi A and Kroemer G: Self-eating and self-killing: Crosstalk between autophagy and apoptosis. Nat Rev Mol Cell Biol 8: 741-752, 2007.

22. Chen YM, Zhou Y, Go G, Marmerstein JT, Kikkawa Y and Miner JH: Laminin beta2 gene missense mutation produces endoplasmic reticulum stress in podocytes. J Am Soc Nephrol 24: 1223-1233, 2013

23. Yu CC, Fornoni A, Weins A, Hakroush S, Maiguel D, Sageshima J, Chen L, Ciancio G, Faridi MH, Behr D, et al: Abatacept in B7 1 positive proteinuric kidney disease. N Engl J Med 369: 2416-2423, 2013.

24. Ma J, Zhang B, Liu S, Xie S, Yang Y, Ma J, Deng Y, Wang W, Xu L, Li R, et al: 1,25-dihydroxyvitamin $\mathrm{D}(3)$ inhibits podocyte uPAR expression and reduces proteinuria. PLoS One 8: e64912, 2013.

25. Zeng C, Fan Y, Wu J, Shi S, Chen Z, Zhong Y, Zhang C, Zen K and Liu Z: Podocyte autophagic activity plays a protective role in renal injury and delays the progression of podocytopathies. J Pathol 234: 203-213, 2014. 
26. Klionsky DJ, Abdalla FC, Abeliovich H, Abraham RT, Acevedo-Arozena A, Adeli K, Agholme L, Agnello M, Agostinis P, Aguirre-Ghiso JA, et al: Guidelines for the use and interpretation of assays for monitoring autophagy. Autophagy 8: 445-544, 2012.

27. Bjorkoy G, Lamark T, Brech A, Outzen H, Perander M, Overvatn A, Stenmark $\mathrm{H}$ and Johansen T: p62/SQSTM1 forms protein aggregates degraded by autophagy and has a protective effect on huntingtin-induced cell death. J Cell Biol 171: 603-614, 2005.

28. Zhou C, Zhong W, Zhou J, Sheng F, Fang Z, Wei Y, Chen Y, Deng X, $\mathrm{Xia} \mathrm{B}$ and Lin J: Monitoring autophagic flux by an improved tandem fluorescent-tagged LC3 (mTagRFP-mWasabi-LC3) reveals that high-dose rapamycin impairs autophagic flux in cancer cells. Autophagy 8: 1215-1226, 2012.
29. Cybulsky AV, Takano T, Papillon J, Kitzler TM and Bijian K: Endoplasmic reticulum stress in glomerular epithelial cell injury. Am J Physiol Renal Physiol 301: F496-F508, 2011.

30. Wu L, Feng Z, Cui S, Hou K, Tang L, Zhou J, Cai G, Xie Y, Hong Q, Fu B and Chen X: Rapamycin upregulates autophagy by inhibiting the mTOR-ULK1 pathway, resulting in reduced podocyte injury. PLoS One 8: e63799, 2013.

31. Seglen PO and Gordon PB: 3-Methyladenine: Specific inhibitor of autophagic/lysosomal protein degradation in isolated rat hepatocytes. Proc Natl Acad Sci USA 79: 1889-1892, 1982.

32. Mackeh R, Perdiz D, Lorin S, Codogno P and Poüs C: Autophagy and microtubules-new story, old players. J Cell Sci 126: 1071-1080, 2013. 